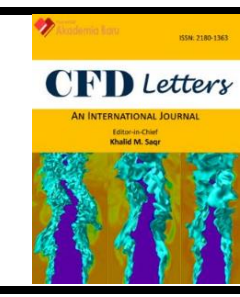

\title{
Computation of Steady Free Convective Boundary Layer Viscous Fluid Flow and Heat Transfer towards the Moving Flat Plate subjected to Suction/Injection Effects
}

\author{
Mohammad Ferdows ${ }^{1}$, Mohammed Shamshuddin ${ }^{2}$, Khairy Zaimi ${ }^{3, *}$ \\ 1 Research Group of Fluid Flow Modeling and Simulation, Department of Applied Mathematics, University of Dhaka, Dhaka-1000, Bangladesh \\ Department of Mathematics, Vaagdevi College of Engineering (Autonomous), Warangal, Telangana, India \\ 3 Boundary Layer Research Group, Institute of Engineering Mathematics, Faculty of Applied and Human Sciences, Universiti Malaysia Perlis, \\ Perlis, Malaysia
}

\section{ARTICLE INFO}

\section{Article history:}

Received 29 October 2020

Received in revised form 1 January 2021

Accepted 9 January 2021

Available online 14 March 2021

Keywords:

Heat transfer; boundary layer flow; moving flat plate; suction/injection

\section{ABSTRACT}

\begin{abstract}
The steady free convective boundary layer viscous fluid flow and heat transfer towards the moving flat plate is investigated. It is important to study the fluid flow and heat transfer problems in the presence of suction and injection effects due to an extensive variety of applications in engineering and industry. Thus, the main objective of the present study is to analyse the impacts of the suction and injection parameter on the velocity and temperature of the fluid as well as the skin friction and the Nusselt number coefficients. The problem has coupled partial differential equations which are converted into ordinary differential equations by employing similarity transformation and adopting of the strong wall suction. The ordinary differential equations thus obtain are handled numerically by utilizing Maple software simulation. The main findings concerning the behaviours of velocity and temperature against various values of the emerging physical parameter such as suction/injection are presented clearly in this numerical examination via graphical illustrations whose physical explanation are discussed thoroughly based on a strong theoretical basis. Furthermore, skin friction and Nusselt number results are studied at different values of pressure gradient. The detail geometry reveals that the velocity and temperature of the fluid decreases with increase of suction/injection parameter. Both skin friction coefficient and Nusselt number decreases with an increase of suction/injection parameter.
\end{abstract}

\section{Introduction}

Fundamental studies of viscous fluids at boundary layer fluid is essential in enhancing systems and device performance, electrical equipment, thermal energy store etc. As a result, the study of viscous boundary layers flows in became prominent. Few studies reported some important results [1-3]. Liquid metal having no Prandtl number (because of very large thermal conductivity) are generally used as coolants and have mechanically significant applications in manufacturing processes such as the cooling of the metallic plate, nuclear reactor etc. Liquid metal has ability to transport heat

\footnotetext{
* Corresponding author.

E-mail address: khairy@unimap.edu.my (Khairy Zaimi)
}

https://doi.org/10.37934/cfdl.13.3.1624 
even if small temperature difference exists between the surface and fluid. This topic has also many applications such as its influence on operating temperatures of power generating and electronic devices.

Newtonian fluid heat transfer characteristics have fascinated the great interest of research community as numerous applications applied by manufacturing and industrial process. In general, the mathematical problems of strong non-linearity and higher order differential equations with viscous fluids because these fluids are unavoidable in many disciplines like manufacture of plastic, pharmaceutical, biotechnological processes. Various constitutive equations were proposed to describe the flow and heat transfer mechanism such as power-law fluid model in which shear stress varies according to power function of strain rate. Several studies on the power-law model have been investigated in the scientific communication by several researchers mathematically. Power-law fluids are implemented to boundary layers by Acrivos et al., [4] and Schowalter [5] and they established the boundary layer conditions for the actuality of similarity solutions. Andersson et al., [6] used exact similarity transformations to examine the flow of an electrically conducting magnetized power-law fluid and extended similar study to thin film power-law fluid considering unsteady flow [7]. NonNewtonian power-law fluid on continuously moving and stretching sheet using Merk-Chao series expansion was studied by Howell et al., [8].

Effects of varying viscosity and thermal conductivity on steady free convective flow and heat transfer along an isothermal vertical plate in the presence of heat sink [9]. Theoretical investigation on boundary layer viscous flow on moving surface with temperature dependent viscosity on moving surface studied Mureithi et al., [10]. The pioneering work on the continuously stretching sheet was first initiated Sakiadis [11]. The problem is extended to discuss the various aspects of flow and heat transfer characteristics. For the case of flow without heat transfer the non-dimensionalized thermal equation depends on the viscosity parameter, dimensionless temperature and the equation depends on the thermal conductivity [12]. PrandtI number and dimensionless temperature Reynolds Number and hence all physical realizations of the related experiment will have the same value of nondimensionalized variables for the same Reynold's Number. It is well known that suction/injection effects play a crucial role in studying heat transfer in several engineering and technology applications. A verity of literature on this topic with different flow feature can be seen in Anuar et al., [13] analyzed the stability analysis to know the impact of suction/injection on stagnation point flow of hybrid nanofluid. Qalta et al., [14] investigated the performance of reciprocating air compressor in unstable air flow in the suction and discharge system and Liang et al., [15] numerically examined aerofoil flow injection at the upper surface. Further studies include [16-22].

This study has been motivated by the work done by Mureithi et al., [10] by considering boundary layer flow over a moving surface in a streaming flow with a temperature dependent dynamic viscosity. By neglecting temperature dependent density used by Mureithi et al., [10], the free convective boundary layer viscous fluid flow and heat transfer towards the moving flat plate with power law reference velocities is considered. The initiation of this work is to highlight the behavior of an incompressible viscous fluid with suction/injection impacts via a moving flat plate. Besides this heat transfer and momentum equations also deliberated. The importance that heat suction/injection effects in different types of fluid flow have been studied by many investigators recently are Bachok et al., [23], Rehman et al., [24] and Li et al., [25]. Since the heat transfer and fluid flow problems with the inclusion of suction and injection effects are very extensive in the industrial applications, this present study able to give a substantial impact on the heat transfer process. In detail, the current analysis has been performed in the presence of suction/injection effects which play important role in the field of engineering such as high temperature analysis, electroplating technologies, and 
metallurgical industries. The governing equations are solved by numerically by utilizing Maple14 (Aziz [26]) software simulation and results is discussed graphically and tabularized.

\section{Model and Formulation}

The system deals with an incompressible, two-dimensional steady flow of fluid flowing past a moving flat plate whose density is $\rho$ over a moving flat surface. The cartesian coordinate system has its origin located at the leading edge with positive $x$-coordinate extending along with the surface in upwards direction, while the $y$ coordinate is measured normal to the flow as shown in Figure 1 . Keeping the origin fixed velocity of the free stream and the flat plate are $U_{e}$ and $U_{p}$ respectively. Suppose that $T_{w}$ be the surface temperature, and the temperature outside the thermal boundary layer is assumed as $T_{\infty}$. Based on the assumptions made above the governing equations ignoring temperature dependent density as considered by Mureithi et al., [10].

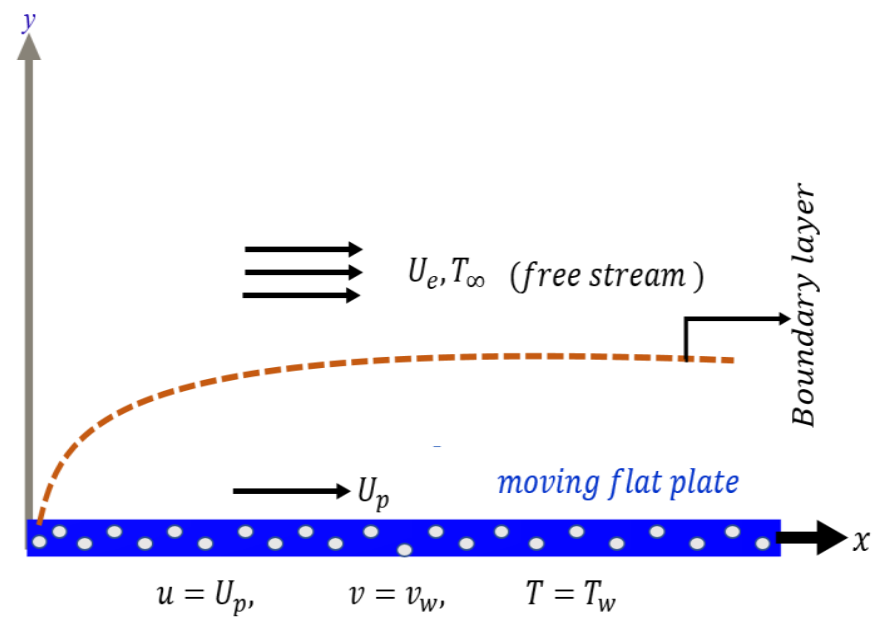

Fig. 1. Flow configuration and schematic model

Continuity equation

$\frac{\partial u}{\partial x}+\frac{\partial v}{\partial y}=0$

Momentum equation

$u \frac{\partial u}{\partial x}+v \frac{\partial v}{\partial y}=U_{e} \frac{d U_{e}}{d x}+v \frac{\partial^{2} u}{\partial y^{2}}$

Energy equation

$u \frac{\partial T}{\partial x}+v \frac{\partial T}{\partial y}=\alpha \frac{\partial^{2} T}{\partial y^{2}}$

The relevant boundary conditions are 


$$
\begin{aligned}
& u=U_{p}(x), \quad v=v_{w}, \quad T=T_{w}, \quad \text { as } y=0 \\
& u=U_{e}, \quad T=T_{\infty} \quad \text { as } y \rightarrow \infty
\end{aligned}
$$

where $u$ and $v$ are the velocity components in the $x$ and $y$ directions respectively. We assume power law variation $U_{p}(x)$ and $U_{e}(x)$ (Mureithi et al., [10]). i.e. $U_{\infty}(x) \sim x^{n}$ and $U_{p}(x) \sim x^{n}$ so that $U_{p}(x)=x^{n} U_{w}$ and $U_{e}(x)=x^{n} U_{\infty}$, where $U_{w}$ and $U_{\infty}$ are constant reference velocities and the pressure gradient $n=\frac{x}{\bar{u}(x)} \frac{d \bar{u}(x)}{d x}$. Therefore $\bar{u}(x)=\left(U_{\infty}+U_{w}\right) x^{n}=B_{o} x^{n}$ where $B_{o}=U_{w}+U_{\infty}$.

\subsection{Transformation}

In this case both the fluid and the surface are moving. This means that we consider the boundary layer flow at a specific given local point. Therefore, we deal with local variables and $\mathrm{n}$ non dimensional terms. We define Reynolds number based on distance $x$ along the wall, hence this local Reynolds numbers is given by $\operatorname{Re}_{x}=x \bar{u}(x) / v$. We must transform the boundary layer equations to a dimensionless system of equations using similarity variable $\eta(x, y)$. It is known that Prandtl boundary layer equations where the free stream velocity varies $U_{e}(x) \propto x^{n}$ normally admit similarity type of solution. We make the following transformation in the boundary layer equations

$u=B_{0} x^{n} f^{\prime}(\eta), \quad \xi=\frac{U_{\infty}}{U_{w}+U_{\infty}}, \quad \theta=\frac{T-T_{\infty}}{T_{w}-T_{\infty}}, \operatorname{Re}_{x}=\frac{B_{0} x^{n} * x}{v}$

The term, $\eta(x, y)$ is a similarity variable and the dimensionless quantity $\mathrm{Re}_{x}$, is the local Reynolds number. By substituting Eq. (5) into Eqs. (2) and (3), we get the following similarity equations results

$n\left[\left(f^{\prime}\right)^{2}-\xi^{2}\right]-\frac{(n+1)}{2} f f^{\prime \prime}=f^{\prime \prime \prime}$,

$\frac{(n+1)}{2} f \theta^{\prime}+\frac{1}{\operatorname{Pr}} \theta^{\prime \prime}=0$

The transformed following boundary conditions Eq. (4) are

$$
\begin{aligned}
& f(0)=f_{w}, \quad f^{\prime}(0)=1-\xi, \quad \theta(0)=1, \text { as } \eta=0 \text {, } \\
& f^{\prime}(\infty)=\xi, \quad \theta(\infty)=0 \text {, as } \eta \rightarrow \infty,
\end{aligned}
$$

where (") prime denotes differentiation with respect to $\eta$. Note that, the skin friction and Nusselt number coefficients are defined as,

$$
\begin{aligned}
& \xi^{2} \sqrt{\operatorname{Re}_{x}} C_{f}=f^{\prime \prime}(0), \\
& N u_{x} / \sqrt{\operatorname{Re}_{x}}=-\theta^{\prime}(0) .
\end{aligned}
$$




\section{Methodology and Validation of Results}

In this paper, the problem of free convective flow of an incompressible fluid past over a moving flat plate is investigated numerically by considering the significant impacts of suction/injection. As described previously the fluid flow model is described mathematically by coupled partial differential equations which are simplified by set of ordinary differential equations by utilizing similarity transformations, resultant non-dimensional ordinary differential equations are simulated numerically with the help of algebra package Maple-14 [26] for outputting graphical and tabular results. The boundary conditions given by Eq.(8) were replaced by using a value of 0.3 for the similarity variable $\eta_{\max }$ as follows

$\eta_{\max }=6, f^{\prime}(6)=\xi, \theta(6)=0$

The choice of $\eta_{\max }=6$ confirms that the far field boundary conditions satisfy correctly. To investigate the flow effects and physical parameters the following considerations are assumed

i. The Prandtl number Pr is chosen 0.72 corresponding to air.

ii. The viscosity parameter $\eta$ is chosen as in Howell et al., [8].

iii. The value of suction or injection parameter $f_{w}$ is chosen arbitrary, value $f_{w}>0$ is suction and $f_{w}<0$ is injection.

For validation of numerical results obtained, we have compared the present results with those obtained by using boundary value problem solver (bvp4c) in MATLAB software as reported by Hale [27]. The bvp4c solver routine is a finite difference code that employs three order Lobatto Illa formula which provides a fourth-order numerical solution.

\section{Results and discussion}

The computed values of skin friction $\left(f^{\prime \prime}(0)\right)$ and Nusselt number $\left(-\theta^{\prime}(0)\right)$ are displayed in Table 1. Table 1 shows the variations of skin friction and Nusselt number with several selected parameters i.e. $f_{w}$ and $n$ for fixed $\operatorname{Pr}=0.72$. Skin friction is strongly increased with increasing $f_{w}$ values at all the pressure gradient values taken in the table. Moreover, it is also observed that local Nusselt number decreases with increasing $f_{w}$ values. We also have compared the present results with another numerical method approach, boundary value problem solver (bvp4c) in MATLAB software. The comparisons are found to be in an excellent agreement as tabulated in Table 2.

Table 1

Variations of $f^{\prime \prime}(0)$ and $-\theta^{\prime}(0)$ with different flow parameters

\begin{tabular}{lllllll}
\hline $\operatorname{Pr}=0.72$ & $n=0.1$ & \multicolumn{5}{c}{$n=1.0$} \\
\cline { 2 - 6 } & $f^{\prime \prime}(0)$ & $-\theta^{\prime}(0)$ & $f^{\prime \prime}(0)$ & $-\theta^{\prime}(0)$ & $f^{\prime \prime}(0)$ & $-\theta^{\prime}(0)$ \\
\hline$f_{w}=-1.2$ & 0.176829 & 0.076778 & 0.530497 & 0.102721 & 0.685852 & 0.101212 \\
$f_{w}=0$ & 0.496614 & 0.332055 & 0.975320 & 0.438538 & 1.232587 & 0.501436 \\
$f_{w}=2.4$ & 1.553684 & 1.116578 & 2.404950 & 1.568867 & 3.005636 & 1.923834 \\
\hline
\end{tabular}


Table 2

\begin{tabular}{|c|c|c|c|c|}
\hline & $f^{\prime \prime}(0)$ & & $-\theta^{\prime}(0)$ & \\
\hline$f_{w}$ & MAPLE 14 & Bvp4c MATLAB & MAPLE 14 & Bvp4c MATLAB \\
\hline-2 & & 0.475810589 & & 0.015708830 \\
\hline-1.2 & 0.685852 & 0.685852481 & 0.101212 & 0.101213318 \\
\hline 0 & 1.232587 & 1.232587624 & 0.501436 & 0.501435934 \\
\hline 2.4 & 3.005636 & 3.005636219 & 1.923834 & 1.923834674 \\
\hline 3 & & 3.526640374 & & 2.326329220 \\
\hline
\end{tabular}

Figures 2 and 3 visualizes the linear velocity and temperature distribution responses to variation in the key control parameter suction/injection (suction: $f_{w}>0$, injection: $f_{w}<0$ ), obtained via MAPLE 14 computational algorithm solutions. Figure 2 illustrates the evolution in velocity profiles for varying $f_{w}, f_{w}=-1.2$ represent the injection and $f_{w}=2.4$ represents the suction which shows the wall is moving faster than the free stream, $\xi=1$ increase in $f_{w}$ results in decrease in the thickness of momentum boundary layer. The presence of suction causes adherence of the boundary layer to plate surface whereas blowing results in the opposite effect. In the case of suction, a markedly lower the linear velocity is computed (see Figure 2). The removal of the fluid via the moving plate destroys the momentum in the boundary layer. Figure 3 shows that the temperature profile reduces with the increase of suction velocity and enhances with the increase of injection velocity. It is worth mentioning that the higher values of injection parameters lead to increase molecular velocity in comparison with the permeable surface $\left(f_{w}=0\right)$ which the linear velocity and temperature of the fluid will be increased.

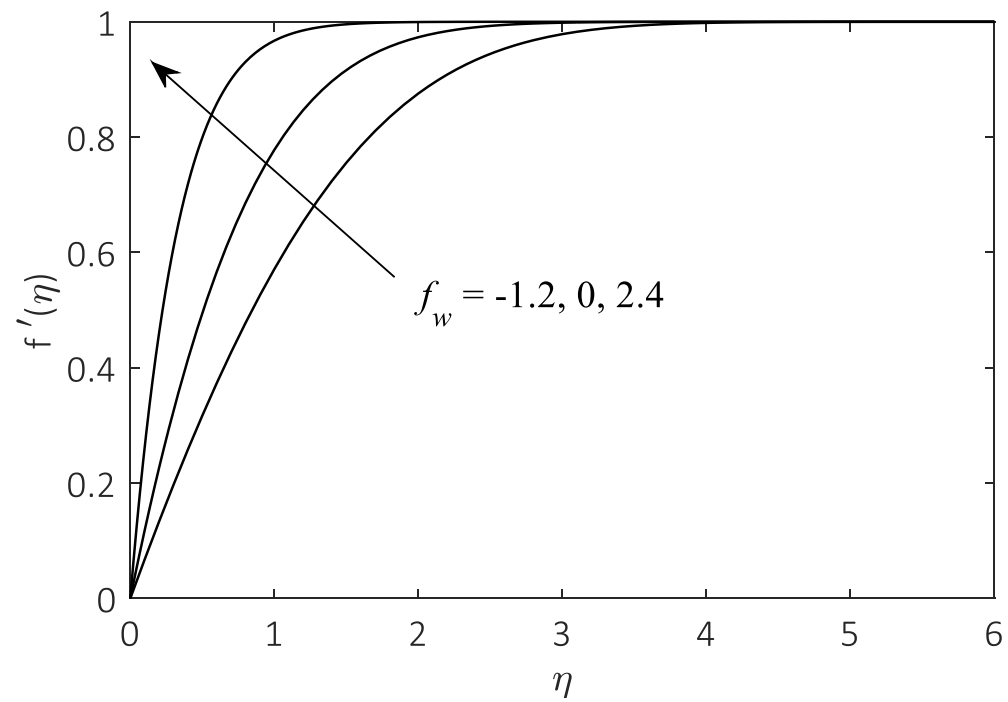

Fig. 2. The dimensionless velocity profile $f^{\prime}(\eta)$ as a factor of $\eta$ for various values of $\xi=1, n=1, \operatorname{Pr}=0.72$ 


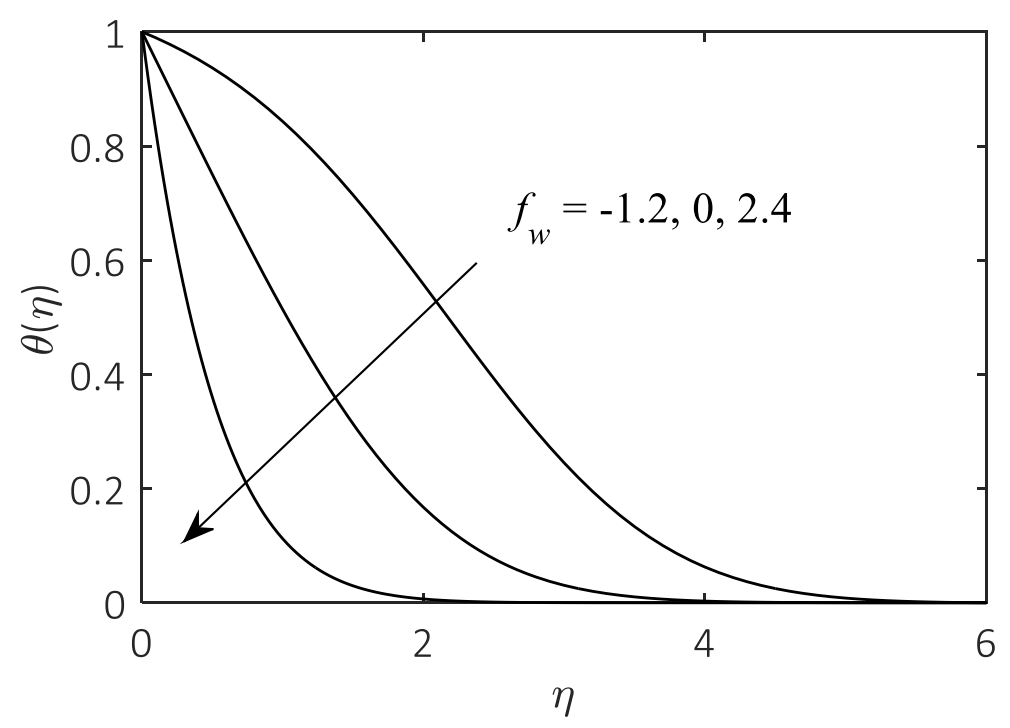

Fig. 3. The dimensionless temperature profile $\theta(\eta)$ as a factor of $\eta$ for various values of $\xi=1, n=1, \operatorname{Pr}=0.72$

\section{Conclusions}

A theoretical study has been presented to investigate steady two-dimensional boundary layer flow over a moving plate with suction/ injection effects. Suction/injection effects has been modelled in boundary conditions at the wall. The wall was assumed to move in the same direction as the free stream hence no reverse flow within the boundary layer. Using scaling variables, we carried out nondimensionlization and order of magnitude analysis of the governing equations. The boundary layer equations were reduced to self-similarity form using the similarity variable $(x, y)$ and by assuming a power law variation on both the free stream and wall velocities i.e. $U_{e}(x) \sim x^{n}$ and $U_{p}(x) \sim x^{n}$. In the case where viscous dissipation was considered, the boundary layer equations only admitted to selfsimilarity solution when the pressure gradient parameter $n=0$. When viscous dissipation was neglected, the flow was similar for all values of $n$. Emerged ordinary differential equations solved numerically with MAPLE 14 computational algorithm. The present simulations show that, the velocity and the temperature of the fluid decrease with the increase of suction or injection. The velocity and the thermal boundary layers thickness decrease with the increase of suction or injection. It is observed that the skin friction coefficient decreases if the value of $f_{w}$ is increased. It is observed that the local Nusselt number decreases if the value of $f_{w}$ is increased. The present simulations have been confined to steady-state flow. Further investigation will consider transient model. It is envisaged that further interest in more realistic fluid flow in different complex geometries are underway and will be communicated imminently.

\section{Acknowledgement}

The author would like to acknowledge the support from the Fundamental Research Grant Scheme (FRGS) under a grant number of FRGS/1/2018/STG06/UNIMAP/02/3 from the Ministry of Education Malaysia.

\section{References}

[1] Messiter, A. F. "Boundary-layer flow near the trailing edge of a flat plate." SIAM Journal on Applied Mathematics 18, no. 1 (1970): 241-257. https://doi.org/10.1137/0118020 
[2] Aydın, Orhan, and Ahmet Kaya. "Laminar boundary layer flow over a horizontal permeable flat plate." Applied Mathematics and Computation 161, no. 1 (2005): 229-240. https://doi.org/10.1016/j.amc.2003.12.021

[3] Vajravelu, K., K. V. Prasad, and Chiu-On Ng. "Unsteady convective boundary layer flow of a viscous fluid at a vertical surface with variable fluid properties." Nonlinear Analysis: Real World Applications 14, no. 1 (2013): $455-464$. https://doi.org/10.1016/j.nonrwa.2012.07.008

[4] Acrivos, Andreas, M. J. Shah, and E. E. Petersen. "Momentum and heat transfer in laminar boundary-layer flows of non-Newtonian fluids past external surfaces." AlChE Journal 6, no. 2 (1960): $312-317$. https://doi.org/10.1002/aic.690060227

[5] Schowalter, William R. "The application of boundary-layer theory to power-law pseudoplastic fluids: Similar solutions." AIChE Journal 6, no. 1 (1960): 24-28. https://doi.org/10.1002/aic.690060105

[6] Andersson, H. I., K. H. Bech, and B. S. Dandapat. "Magnetohydrodynamic flow of a power-law fluid over a stretching sheet." International Journal of Non-Linear Mechanics 27, no. 6 (1992): 929-936. https://doi.org/10.1016/00207462(92)90045-9

[7] Andersson, H. I., J. B. Aarseth, N. Braud, and B. S. Dandapat. "Flow of a power-law fluid film on an unsteady stretching surface." Journal of Non-Newtonian Fluid Mechanics 62, no. 1 (1996): 1-8. https://doi.org/10.1016/0377-0257(95)01392-X

[8] Howell, T. G., D. R. Jeng, and K. J. De Witt. "Momentum and heat transfer on a continuous moving surface in a power law fluid." International Journal of Heat and Mass Transfer 40, no. 8 (1997): 1853-1861. https://doi.org/10.1016/S0017-9310(96)00247-5

[9] Mahanti, N. C., and Pramod Gaur. "Effects of varying viscosity and thermal conductivity on steady free convective flow and heat transfer along an isothermal vertical plate in the presence of heat sink." (2009): 23-28.

[10] Mureithi, E.W., J.J. Mwaonanji, and O.D. Makinde. "On the boundary layer flow over a moving surface in a fluid with temperature dependent viscosity." Open Journal of Fluid Dynamics 3 (2013): 135-140. https://doi.org/10.4236/ojfd.2013.32017

[11] Sakiadis, B.C "Boundary layer behaviour on continious solid surfaces." AlChE Journal 7 (1961):26-28. https://doi.org/10.1002/aic.690070108

[12] Arunachalam, M, and N.R. Rajappa. "Forced convection in liquid metals with variable thermal conductivity and capacity." Acta Mechanica 31 (1978): 25-31. https://doi.org/10.1007/BF01261185

[13] Anuar, N.S., N. Bachok, N.M. Arifin, and H. Rosali. "Effect of suction/injection on stagnation point flow of hybrid nanofluid over an exponentially shrinking sheet with stability analysis." CFD Letters 11, no. 12 (2019): 21-33.

[14] Qalta, H.J., A.S. Mahmood, and L.J. Habeeb. "Study the effect of unstable air flow in the suction and discharge system on the performance of reciprocating air compressor." Journal of Advanced Research in Fluid Mechanics and Thermal Sciences 65, no. 1 (2020): 54-71.

[15] Liang, Chua Bing, Akmal Nizam Mohammed, Azwan Sapit, Mohd Azahari Razali, Mohd Faisal Hushim, Amir Khalid, and Nurul Farhana Mohd Yusof. "Numerical Simulation of Aerofoil with Flow Injection at the Upper Surface." CFD Letters 12, no. 1 (2020): 98-110.

[16] Himanshu, U., A.K. Pandey, M. Kumar. "MHD flow of Ag-water nanofluid over a flat porous plate with viscous ohmic dissipation, suction/injection and heat generation/absorption." Alexandria Engineering Journal 57, no. 3 (2018): 1839-1847. https://doi.org/10.1016/j.aej.2017.03.018.

[17] Mishra, A.K., A.K. Pandey, and M. Kumar. "Ohmic -viscous dissipation and slip effects on nanofluid flow over a stretching cylinder with suction/injection." Nanoscience and Technology An International Journal 9, no. 2 (2018):99115. https://doi.org/10.1615/NanoSciTechnollntJ.2018025410

[18] Himanshu, U., A.K. Pandey, M. Kumar. "Thermophoresis and suction/injection roles on free convective MHD flow of Ag-kerosene oil nanofluid." Journal of Computational Design and Engineering 7, no.3 (2020): 386-396. https://doi.org/10.1093/jcde/qwaa031.

[19] Himanshu, U., A.K. Pandey, M. Kumar. "Ohmic heating and non-uniform heat source/sink roles on 3D DarcyForchheimer flow of CNTs nanofluids over a stretching surface." Arabian Journal of Science and Engineering (2020). https://doi.org/10.1007/s13369-020-04826-7.

[20] Nagaraju, G., G. Mahesh. "Magnetohydrodynamic viscous fluid flow and heat transfer in a circular pipe under an externally applied constant suction." Heliyon 5, no. 2 (2019): e01281 https://doi.org/10.1016/i.helivon.2019.e01281

[21] Ramana Murthy, J.V., G. Nagaraju. "The flow of a couple stress fluid generated by a circular cylinder subjected to longitudinal and torsional oscillations." Contemporary Engineering Sciences 2, no. 10 (2009) $451-461$. https://doi.org/10.12988/ces

[22] Ramana Murthy, J.V., G. Nagaraju, K.S. Sai. "Numerical solution for MHD flow of micropolar fluid between two concentric rotating cylinders with porous lining." International Journal of Nonlinear Science 13, no.2 (2012): 183193. 
[23] Bachok, N., A. Ishak, and I. Pop. "Boundary layer flow over a moving surface in a nanofluid with suction or injection." Acta Mechanica Sinica 28 (2012): 34-40. https://doi.org/10.1007/s10409-012-0014-x

[24] Rehman, S., M. Idress., R.A. Shah, and Z. Khan. "Suction/injection effects on an unsteady MHD Casson thin film flow with slip and uniform thickness over a stretching sheet along variable flow properties." Boundary Value Problems 2019 (2019): Article ID: 26. https://doi.org/10.1186/s13661-019-1133-0.

[25] Li, Botong, Yikai Yang, and Xuehui Chen. "A power-law liquid food flowing through an uneven channel with nonuniform suction/injection." International Journal of Heat and Mass Transfer 144 (2019): 118639. https://doi.org/10.1016/j.ijheatmasstransfer.2019.118639

[26] Aziz, Abdul. Heat conduction with maple. Philadelphia: RT Edwards, 2006.

[27] Hale, N. P. "A sixth-order extension to the matlab bvp4c software of j. kierzenka and I. shampine." Department of Mathematics, Imperial College London (2006). 